\title{
细胞色素 $\boldsymbol{b}$ 和 12S rRNA 基因序列变异 与东方鲀属鱼类系统发育
}

\author{
张玉波 ${ }^{\circledR(}$ 何舜平 $^{\mathbb{N}^{*}}$ \\ (1) 中国科学院水生生物研究所, 武汉 430072; (2) 中国科学院研究生院, 北京 100049.*联系人, E-mail: clad@ihb.ac.cn)
}

\begin{abstract}
摘要 东方鲀属鱼类是一个区域性分布类群, 该属目前包括 22 个有效种, 主要分布区域从日本海西部 到中国沿海. 本研究通过联合 17 种(21 尾)东方鲀属鱼类的细胞色素 $b$ (1137 bp)和 12S rRNA (952 bp)基 因全序列研究了东方鲀属鱼类的系统发育关系. 采用了邻接法 $(\mathrm{NJ}) 、$ 最大简约法 $(\mathrm{MP}) 、$ 最大似然法(ML) 和 Baysian 方法构建了分子系统树. 结果表明: ( i ) 东方鲀属鱼类为一单系类群; (ii) 基于分子系统发 育分析, 东方鲀属鱼类可划分为 6 个亚群; (iii) 在分子水平上, 本属各鱼类物种的遗传距离比较接近, 这显示了其物种间分化时间较短. 研究结果还表明, 本属鱼类物种分类情况可能还需要进一步的澄清. 研究中还发现种间杂交现象可能对本属鱼类的物种分化过程有重要影响.
\end{abstract}

关键词 系统发育 细胞色素b 12S rRNA 东方鲀属鱼类

鲀形目(Tetraodontiformes)鱼类是一较大类群, 大约有 350 种, 该类群形态变化大, 广泛分布于热带 和亚热带海域 11 . 而东方鲀属 (Takifugu Abe)鱼类则是 隶属于鲀形目鱼类的一个区域性分布类群, 主要生 活在东亚近海及咸淡水区域, 只有极少数种类生活 在深海, 主要分布区域从日本岛西部到中国沿海, 极 少数可以分布到印度洋 ${ }^{[2-4]}$ (图 1). 据中国动物志记载, 我国有 22 种东方鲀属鱼类(其中包括两个新种，而 fishbase 数据库中(http://www.fishbase.org)记载的有 效种名为 23 个), 分布在我国的一些大的河口区域 (如珠江、长江等河口)和渤海、黄海、东海及南海等

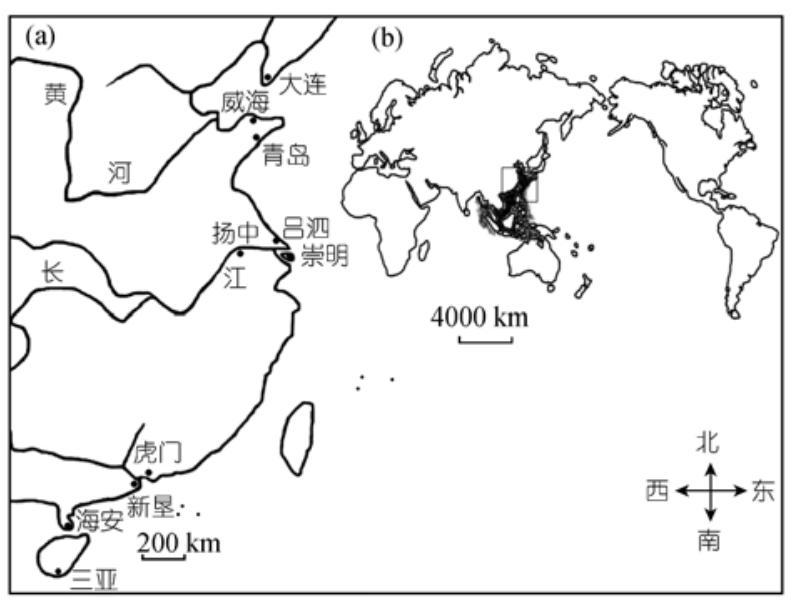

图 1 东方鲀属鱼类的采样点(a)及分布(b，阴影部分)简图
海域 ${ }^{[4]}$.

该属鱼类因含有河豚毒素, 且具有较高经济价 值, 一直被研究者予以关注. 从 20 世纪 90 年代开始, 红鯺东方鲀拥有的“简约”基因组已逐渐成为后基因组 时代的一个新研究热点, 应用全基因组“乌枪法”作出 的覆盖基因组 $95 \%$ 的草图已经完成 ${ }^{[5]}$, 可以预见东方 鲀属鱼类在未来基因查询、注释和功能研究过程中将 发挥非常重要的作用 ${ }^{[4,6,7]} .20$ 世纪 50 年代, 东方鲀属 才被作为一个独立的分类单元, 从圆鲀属(Sphoeroides) 划分出来 ${ }^{[8 \sim 11]}$.

一直以来, 鲀形目鱼类的分子系统发育研究主 要集中在属间及属以上分类单元 ${ }^{[12,13]}$, 而能够说明 有关该类群种间或种内的分化及其进化趋势的研究 还相对比较缺乏 ${ }^{[14]}$, 有关属内系统发育关系的研究 主要集中在形态层面上 ${ }^{[8 \sim 11,15,16]} .1949$ 年, $\mathrm{Abe}^{[9]}$ 指出 生活在中国和日本近海的该属鱼类可分为 6 个亚属 计 18 个种. 我国的鱼类学家成庆泰等人 ${ }^{[15]}$ 在 1975 年 系统地研究了分布于我国的 15 种(含两个新种)东方 鲀属鱼类的分类及种间关系. 在细胞学层面上, 王可 玲等人 ${ }^{[16]}$ 利用肌浆蛋白多态性研究了 13 种东方鲀属 鱼类间的相互关系. Miyaki 等人 ${ }^{[17]}$ 做了 6 种东方鲀属 鱼类的核型比较分析.

近些年来, 基于 DNA 序列的分子系统学方法开 始被逐渐引入到东方鲀属鱼类的系统发育研究中, 如

2007-02-27 收稿, 2007-09-25 接受

国家自然科学基金(批准号: 30225008)资助项目 
陈超等人 ${ }^{[18]}$ 利用 RAPD (random amplified polymorphic DNA)研究了假睛东方鲀 (T. pseudommus)、暗纹东方 鲀(T. fasciatus) 和分别产自日本和中国的红鯺东方鲀 (T. rubripes)之间的系统发育关系. Song 等人 ${ }^{[4,19]}$ 利用 RAPD 和 $16 \mathrm{~S}$ rRNA (572 bp)研究了假睛东方鲀、红鯺 东方鲀、虫纹东方鲀 (T. vermicularis)、黄鯺东方鲀 (T. xanthopterus) 和星点东方鲀( T. niphobles) 等 5 种东方 鲀属鱼类的系统发育关系. Song 等人 ${ }^{[201}$ 研究了假睛 东方鲀和红鯺东方鲀的群体遗传结构等. 而较为系 统的东方鲀属鱼类内部系统发育关系研究仍比较缺 $乏^{[4,18 \sim 20]}$.

本文的主要目的是研究东方鲀属鱼类的系统发 育关系，探讨该类群的单系性和评估当前该类群鱼 类中现有物种分类情况. 线粒体基因经常被用来作 为研究近缘物种系统发育关系的分子标记, 其中 细胞色素 $b$ 更由于是蛋白质编码基因 ${ }^{[21]}$, 同时序列中 含有序列变异较快的区域和较慢的密码子位点而 被广泛认为是研究系统发育关系的主要分子标记之 一 2224$]$. 本研究中利用线粒体基因细胞色素 $b$ 和 $12 \mathrm{~S}$
rRNA 基因序列来研究东方鲀属鱼类的系统发育关系, 同时根据 Holcroft ${ }^{[12,13]}$ 的研究结果, 结合实际采样情 况，选用了黑青斑河豚(Tetraodon nigroviridis)和克氏 兔头鲀 (Lagocephalus gloveri) 作为本研究的外类群.

\section{1 材料与方法}

（i ）标本采集与鉴定．近年来，东方鲀属鱼类 种群数量下降得很快 ${ }^{[25]}$, 少部分东方鲀属鱼类难以 获得实验标本. 研究中大约涵盖了超过该属鱼类四 分之三的有效种(表 1), 没有包括紫色东方鲀 (T. por phyreus)、豹纹东方鲀 (T. pardalis)、网纹东方鲀 ( $T$. reticularis)、斑带东方鲀( $T$. guttulatus) 和斜斑东方鲀 ( $T$. plagiocellatus)等 5 种东方鲀属鱼类. 所有的物种鉴定 均依照中国动物志 ${ }^{[26]}$ 种的分类标准进行, 标本用 95\%的酒精进行固定, 保存于中国科学院水生生物研 究所淡水鱼类博物馆.

(ii ) DNA 提取、扩增和测序. 基因组 DNA 提 取是以 $95 \%$ 乙醇保存的鯺条样本为实验材料, 采用 标准的苯酚/氯仿抽提法提取基因组 DNA 样本 ${ }^{[27]}$, 用

表 1 东方鲀属鱼类代表种类及相关信息

\begin{tabular}{|c|c|c|c|c|}
\hline \multirow{2}{*}{ 种类 } & \multirow{2}{*}{ 收录号 } & \multirow{2}{*}{ 收集地 } & \multicolumn{2}{|c|}{ GenBank 登录号 } \\
\hline & & & 细胞色素 $b$ & $12 \mathrm{~S}$ rRNA \\
\hline Tetraodon nigroviridis & & & DQ019313 & DQ019313 \\
\hline Lagocephalus gloveri & IHB0411227 & 山东青岛 & EF126108 & EF126128 \\
\hline T. poecilonotus & & & AY267360 & \\
\hline T. oblongus & IHB0411173 & 海南三亚 & EF126107 & EF126110 \\
\hline T. niphobles & IHB0411175 & 海南三亚 & EF126106 & EF126109 \\
\hline T. alboplumbeus & IHB0411177 & 海南三亚 & EF126097 & EF126111 \\
\hline T. ocellatus & IHB0411191 & 广东虎门 & EF126095 & EF126113 \\
\hline T. variomaculatus & IHB0411195 & 广东虎门 & EF126096 & EF126114 \\
\hline T. orbimaculatus & IHB0411198 & 广东虎门 & EF126100 & EF126122 \\
\hline T. orbimaculatus & IHB0411207 & 广东新晆 & EF126104 & EF126126 \\
\hline T. fasciatus & IHB0411203 & 江苏扬中 & EF126102 & EF126123 \\
\hline T. fasciatus & IHB0411203 & 广东新圼 & EF126101 & EF126124 \\
\hline T. flavidus & IHB0411217 & 江苏扬中 & EF126093 & EF126117 \\
\hline T. flavidus & & 江苏吕泗 & EF126092 & EF126116 \\
\hline T. coronoidus & IHB0411223 & 江苏吕泗 & EF126103 & EF126125 \\
\hline T. xanthopterus & IHB0411224 & 山东青岛 & EF126099 & EF126115 \\
\hline T. rubripes & IHB0411226 & 江苏吕泗 & EF126089 & EF126119 \\
\hline T. rubripes & & & AJ421455 & AJ421455 \\
\hline T. basilevskianus & IHB0507170 & 山东威海 & EF126091 & EF126121 \\
\hline T. stictonotus & IHB0507172 & 山东威海 & EF126098 & EF126112 \\
\hline T. vermicularis & & 辽宁大连 & EF126105 & EF126127 \\
\hline T. pseudommus & IHB0507101 & 辽宁大连 & EF126090 & EF126120 \\
\hline T. bimaculatus & IHB0411214 & 上海崇明 & EF126094 & EF126118 \\
\hline
\end{tabular}


于 $\mathrm{PCR}$ 扩增. 引物设计参考了 Xiao 等人 ${ }^{[28]}$ 和刘焕 章 ${ }^{[29]}$ 的报道，并根据已经发表的红鯺东方鲀的线粒 体基因组序列 ${ }^{[7]}$ 进行了比对修正. 细胞色素 $b$ 和 $12 \mathrm{~S}$ rRNA 序列的 PCR 扩增和测序引物均为细胞色素 $b \mathrm{~F}$ (5'-GGCGTGAAAAACCATCGTTG-3')、细胞色素 $b$ R (5'-CCCCGACATTCGGTTTACAAGAC-3') 和 $12 \mathrm{~s}$ F (5'GCAGAGTACTGAAGATGCTAAG-3')及 12s R (5'-CGTCAACTCGGTGTAAGG-3'). $60 \mu \mathrm{L}$ 的 PCR 反应体系 中含有约 $50 \mathrm{ng}$ 的模板 DNA, 10×缓冲液 $5 \mu \mathrm{L}, 2.5$ $\mathrm{mmol} / \mathrm{L} \mathrm{dNTP}$ 各 $2 \mu \mathrm{L}$, 引物 $(10 \mathrm{pmol} / \mathrm{L})$ 各 $2.5 \mu \mathrm{L}, \mathrm{Taq}$ 聚合酶 $2.0 \mathrm{U}$, 最后补足灭菌双蒸水至终体积. PCR 反 应条件为: $94^{\circ} \mathrm{C}$ 预变性 $4 \mathrm{~min} ; 94^{\circ} \mathrm{C}$ 变性 $45 \mathrm{~s}, 52^{\circ} \mathrm{C}$ 退火 $45 \mathrm{~s}, 72^{\circ} \mathrm{C}$ 延伸 $1 \mathrm{~min}, 35$ 个循环; $72^{\circ} \mathrm{C}$ 延伸 $8 \mathrm{~min}$. 每 次反应设立不含 DNA 模板的空白对照组. 扩增产物 经 $0.8 \%$ 的琼脂糖凝胶电泳后检测, 利用 Glassmilk 纯 化试剂盒(BioStar)回收进行 DNA 测序(序列 GenBank 登录号见表 1). 黑青斑河豚的细胞色素 $b$ 和 $12 \mathrm{~S}$ rRNA 的基因序列从 GenBank 中下载(登录号为: DQ019313).

(iii) 序列与系统发育分析. 使用 DNAstar 中 Editseq 模块 (http://www.dnastar.com/web/index.php) 进行序列拼接. 分别采用 Clustal $\mathrm{X}^{[30]}$ 和 SEAVIEW ${ }^{[31]}$ 软件进行序列排序和序列分析，排序的最终结果被 保存为 NEXUS 格式的文件. 已发表的红鯺东方鲀的 细胞色素 $b$ 基因和 12S rRNA 基因序列(登录号: AJ421455)作为序列排定的模板序列，它们同时也被 包括在随后的系统发育分析中.

据 Wiens ${ }^{[32]}$ 的建议, 除非缺失的数据非常多, 否 则不完全数据的增加可能改善系统发育的准确性, 所 以从 GenBank 中下载的斑点东方鲀 (T. poecilonotus) 的 $424 \mathrm{bp}$ 的细胞色素 $b$ 基因序列也被用于本属鱼类的系 统发育研究, 它所对应的其他部分被作为缺失碱基. 因此, 本研究中用于系统发育关系研究的细胞色素 $b$ 基因序列共计有 17 种东方鲀属鱼类，而用于分析的 $12 \mathrm{~S}$ rRNA 基因序列则只有 16 种该属鱼类. 此外, 两 种外类群鱼 Tetraodon nigroviridis 和 Lagocephalus glover $i$ 的细胞色素 $b$ 和 $12 \mathrm{~S}$ rRNA 基因序列也一起被 用于系统发育研究中(表 1).

细胞色素 $b$ 和 $12 \mathrm{~S}$ rRNA 基因序列的系统发育相 容性分析 (phylogenetic congruence) 通过 PAUP* $4.0 \mathrm{~b} 10^{[33]}$ 软件中的隔离同质检验 (partition homogeneity test)完成 ${ }^{[34]}$. 检验结果支持两序列联合进行系统发育
分析 $(P=0.962) . \mathrm{DAMBE}^{[35]}$ 软件被用来作单个基因 序列、细胞色素 $b$ 的第 3 位点和联合序列的饱和性分 析(不包括外类群), 进化模型选用是 TN93 $3^{[36]}$. 遗传 距离 $\left(P\right.$-distance) 及转换/颠换的计算在 MEGA3 $3^{[37]}$ 软 件中完成. 碱基频率、碱基偏倚(com- positional bias) 等分析在 PAUP* $4.0 \mathrm{~b} 10^{[33]}$ 中完成. 系统发育分析在 PAUP* $4.0 \mathrm{~b} 10^{[33]}$ 软件包和 MrBayes 3.04b $\mathrm{b}^{[38]}$ 软件中进 行, 分别采用了邻接法 (neighbor-joining distance, NJ)、最大简约法(maximum parsimony, MP) ${ }^{[39]}$ 、最大似 然法 (maximum likelihood, ML) ${ }^{[40]}$ 和 贝 叶 斯 (Bayesian) ${ }^{[41]}$ 法来构建系统发育树，其中贝叶斯树是 在 MrBayes 3.04b $\mathrm{b}^{[38]}$ 中完成的. ML, MP 和 NJ 分析中 均选取了启发式搜索(heuristic searches)和树二等分 再连接(tree-bisection reconnection, TBR)进行构树, 并采用自展值分析(bootstraps, BS) ${ }^{[42]}$ 进行检验, 分别 对应 250, 1000 和 1000 随机重复抽样; 贝叶斯法中节 点支持率为后验概率 (posterior probabilities, PP). ML 树和 NJ 树中替代模型的选择采用 Modltest 3.06 $6^{[43]}$ 程 序进行分析, 按 hLRTs 标准, 最适合的替代模型为 GTR + G + I (GTR = General Time Reversible model; G = gamma distributed site-to-site variation; $\mathrm{I}=$ proportion of invariable sites $)^{[43,44]}$. MP 分析中采用加权分析, 细 胞色素 $b$ 基因序列的第 3 位点 $T \mathrm{v} / T \mathrm{i}$ (transversion/ transition)值为 $T \mathrm{v}: T \mathrm{i}=8: 1$, 被用于 MP 分析中，而 联合序列的其他部分采用的是相同的权重. 贝叶斯 法分析中也采用了混合模型分析, 模型的选择是针 对细胞色素 $b$ 基因的不同位点和 $12 \mathrm{~S} \mathrm{rRNA}$ 基因的, 在 MODELTEST 3.06 年3]软件中完成的. 利用 PAUP* $4.0 \mathrm{~b} 10$ 中的 two-tailed Wilcoxon signed-ranks test ${ }^{[45]}$ 和 Shimodaira-Hasegawa(SH)检验 ${ }^{[46]}$ ，检验不同构树方 法所得到的系统发育树之间是否收敛, 来帮助选择 最优的系统发育树. 为了检验是否偏离分子钟, 将有 分子钟约束的 ML 树与不受分子钟约束的 ML 树的 似然对数值相比较 (likelihood-ratio test, LRT), 如果 二者没有显著差异，则分子钟不被拒绝 ${ }^{[38]}$.

\section{2 结果}

\section{1 序列分析}

用于联合分析的序列共包括了 19 种 23 个个体 (包括外类群)，序列长度为 $2089 \mathrm{bp}$ ，其中包括 1137

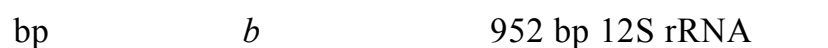
序列. 从获得的序列数据矩阵上看, 东方鲀属鱼类的 序列相似性较高, 特别体现在 $12 \mathrm{~S}$ rRNA 基因序列上. 
细胞色素 $b$ 基因序列编码 378 个氨基酸, 以 ATG 作 为起始密码子, $\mathrm{AAG}$ 作为终止密码子, 同外类群中的 黑青斑河豚序列比对分析发现, 较东方鲀属鱼类有 2 个缺失位点(计 $6 \mathrm{bp}$ ). 同时细胞色素 $b$ 基因序列显示 出较明显的碱基偏倚, 这也是线粒体基因的一个显 著特征 ${ }^{[47]}$, 其中 $\mathrm{G}$ 的含量较低, 全序列中仅占有 $14.4 \%$, 而第 3 位点仅为 $6.8 \%$, 这同以前报道的辐鯺 亚纲 (Actinopterygii) 鱼类细胞色素 $b$ 基因序列类 似 ${ }^{[48,49]}$. 从密码子上面看, 第 3 位点表现出最强的偏 倚 $(0.33)$, 其次是第 1 位点 $(0.20)$, 而第 2 位点基本观 察不到什么偏倚 $(0.02)$, 这同 Peng 等人 ${ }^{[24]}$ 的结果一致. 碱基组成的同质性检验结果包括密码子各位点及全 部位点的检验, 细胞色素 $b$ 基因: $\chi^{2}=23.31, \mathrm{~d} f=69$, $P=1.00$, 第 1 位点: $\chi^{2}=8.56, \mathrm{~d} f=69, P=1.00$; 第 2 位点: $\chi^{2}=8.73, \mathrm{~d} f=69, P=1.00$; 第 3 位点: $\chi^{2}=$ $51.04, \mathrm{~d} f=69, P=0.95$; 表明碱基组成是同质的. $12 \mathrm{~S}$ rRNA 基因序列的碱基平均含量同刘焕章 ${ }^{[29]}$ 有关鲤 形目鱼类报道的结果一致, 序列同质性检验结果: $\chi^{2}$ $=7.46, \mathrm{~d} f=66, P=1.00$. 序列饱和性分析表明不同 序列和不同位点间突变没有达到饱和, 可用于系统 发育分析(图 2). 表 2 给出了基于细胞色素 $b$ 基因序
列的遗传 $P$ 距离序列分歧矩阵, 东方鲀属鱼类的遗传 距离在 0 (圆斑东方鲀 $(T$. orbimaculatus) 和暗纹东方 鲀(T. fasciatus))到 0.079 (横纹东方鲀(T. oblongus) 和 密点东方鲀 $(T$. stictonotus $)) 之$ 间, 内类群同外类群之 间的遗传距离在 0.189 (黑青斑河豚和双斑东方鲀 $(T$ bimaculatus))到 0.259 (克氏兔头鲀和密点东方鲀)之 间.

\section{2 系统发育关系}

本研究中不同分析方法所得到的系统发育树的 拓扑结构基本上是一致的，各种分析方法所得的结 果均表明东方鲀属鱼类系一单系类群. Bayesian, MP 和 NJ 方法所获得的节点支持率分别为 $P P=100, B S=$ 100 和 $\mathrm{BS}=100$. 图 3 给出的是一棵 ML 树 $(-\ln L=$ 6627.22), 它支持了该属鱼类是一个单系类群 $(\mathrm{BS}=$ 99)(图 3); 其他方法分析也可以得到类似的结果(结 果末显示). 通过 ML 树, 我们可以了解到, 在东方鲀 属鱼类中形成 6 个比较大的类群: A 支中，弓斑东方 鲀 (T. ocellatus) 和花斑东方鲀 ( $T$. variomaculatus $)$ 构成 姐妹群关系 $(\mathrm{BS}=100) ; \mathrm{B}$ 中包括有 4 个物种: 斑点 东方鲀 (T. poecilonotus)、黄鯺东方鲀 (T. xanthopterus)、铅点东方鲀(T. alboplumbeus)和密点东方鲀 $(T$.

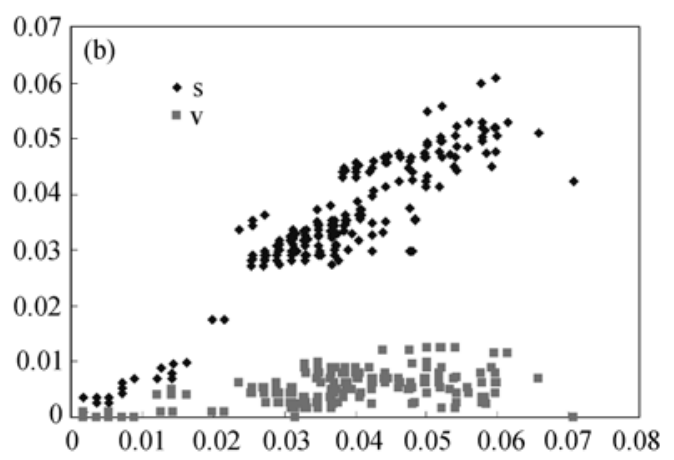

$\begin{array}{lllllllll}0 & 0.01 & 0.02 & 0.03 & 0.04 & 0.05 & 0.06 & 0.07 & 0.08\end{array}$
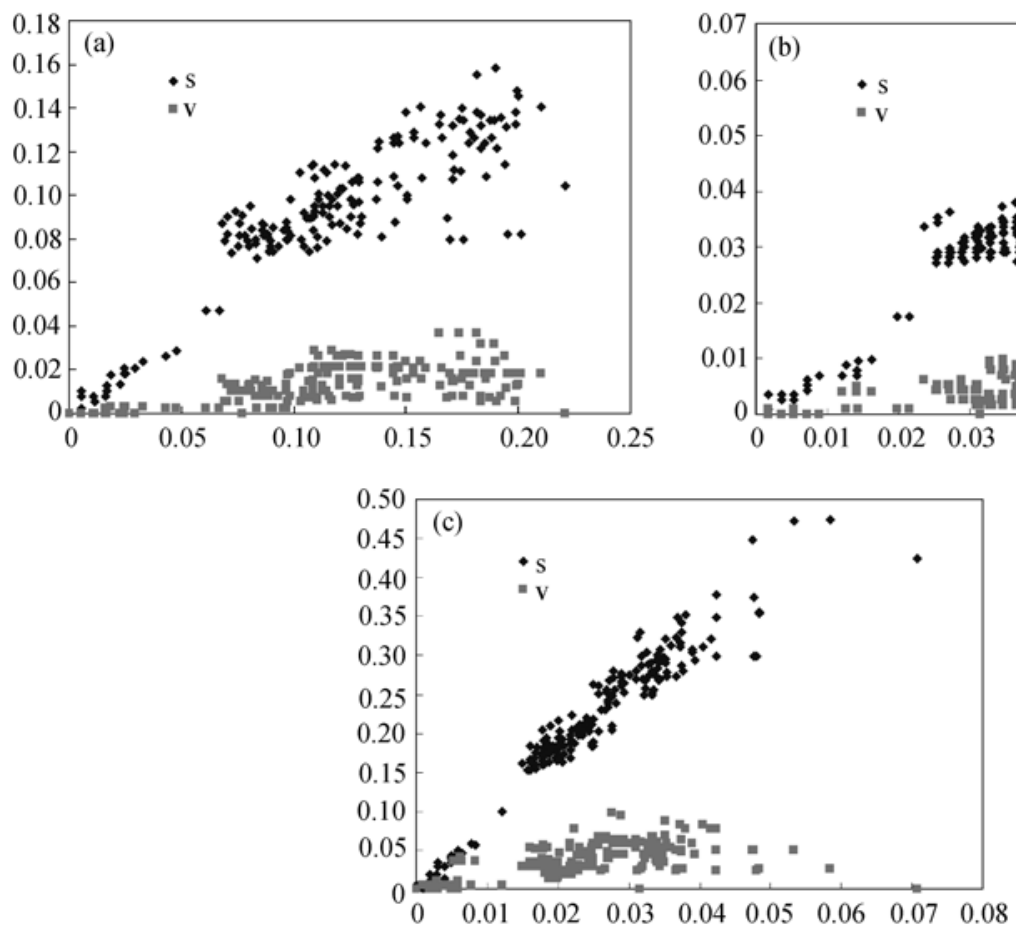

图 2 饱和性检验结果

(a) 细胞色素 $b$ 基因第 3 位点检验结果; (b) 细胞色素 $b$ 基因全序列结果; (c) 联合序列结果. 采用 TN93 距离, 含 21 个条序列(不包括外类群). $\mathrm{s}$ 表示转换; $\mathrm{v}$ 表示颠换 
表 2 本研究中 19 个类群细胞色素 $b$ 基因序列的 $p$-distance 矩阵的遗传距离

\begin{tabular}{|c|c|c|c|c|c|c|c|c|c|c|c|c|c|c|c|c|c|c|}
\hline & 1 & 2 & 3 & 4 & 5 & 6 & 7 & 8 & 9 & 10 & 11 & 12 & 13 & 14 & 15 & 16 & 17 & 18 \\
\hline \multicolumn{19}{|l|}{ T. rubripes } \\
\hline T. pseudommus & 0.003 & & & & & & & & & & & & & & & & & \\
\hline T. stictonotus & 0.005 & 0.003 & & & & & & & & & & & & & & & & \\
\hline T. flavidus & 0.034 & 0.037 & 0.04 & & & & & & & & & & & & & & & \\
\hline T. bimaculatus & 0.032 & 0.034 & 0.037 & 0.018 & & & & & & & & & & & & & & \\
\hline T. ocellatus & 0.042 & 0.045 & 0.042 & 0.034 & 0.042 & & & & & & & & & & & & & \\
\hline T. variomaculatus & 0.042 & 0.045 & 0.042 & 0.034 & 0.042 & 0 & & & & & & & & & & & & \\
\hline T. alboplumbeus & 0.045 & 0.048 & 0.051 & 0.048 & 0.051 & 0.042 & 0.042 & & & & & & & & & & & \\
\hline T. stictonotus & 0.056 & 0.059 & 0.062 & 0.042 & 0.051 & 0.051 & 0.051 & 0.059 & & & & & & & & & & \\
\hline T. xanthopterus & 0.031 & 0.034 & 0.037 & 0.029 & 0.032 & 0.037 & 0.037 & 0.043 & 0.051 & & & & & & & & & \\
\hline T. orbimaculatus & 0.026 & 0.029 & 0.032 & 0.035 & 0.032 & 0.032 & 0.032 & 0.034 & 0.051 & 0.026 & & & & & & & & \\
\hline T. fasciatus & 0.026 & 0.029 & 0.032 & 0.037 & 0.034 & 0.031 & 0.031 & 0.034 & 0.056 & 0.026 & 0 & & & & & & & \\
\hline T. coronoidus & 0.026 & 0.029 & 0.032 & 0.037 & 0.034 & 0.031 & 0.031 & 0.034 & 0.056 & 0.026 & 0 & 0 & & & & & & \\
\hline T. vermicularis & 0.032 & 0.034 & 0.037 & 0.037 & 0.034 & 0.034 & 0.034 & 0.034 & 0.056 & 0.037 & 0.016 & 0.016 & 0.016 & & & & & \\
\hline T. niphobles & 0.051 & 0.054 & 0.056 & 0.054 & 0.051 & 0.056 & 0.056 & 0.059 & 0.076 & 0.062 & 0.037 & 0.043 & 0.042 & 0.048 & & & & \\
\hline T. oblongus & 0.054 & 0.056 & 0.059 & 0.057 & 0.054 & 0.059 & 0.059 & 0.062 & 0.079 & 0.065 & 0.04 & 0.045 & 0.045 & 0.051 & 0.003 & & & \\
\hline T. poecilonotus & 0.04 & 0.042 & 0.045 & 0.037 & 0.034 & 0.04 & 0.04 & 0.045 & 0.054 & 0.034 & 0.029 & 0.034 & 0.034 & 0.034 & 0.054 & 0.056 & & \\
\hline Tetraodon nigroviridis & 0.199 & 0.196 & 0.199 & 0.186 & 0.189 & 0.216 & 0.216 & 0.195 & 0.203 & 0.195 & 0.191 & 0.196 & 0.196 & 0.206 & 0.209 & 0.206 & 0.192 & \\
\hline Lagocephalus gloveri & 0.234 & 0.237 & 0.241 & 0.23 & 0.233 & 0.233 & 0.233 & 0.244 & 0.259 & 0.256 & 0.24 & 0.245 & 0.245 & 0.245 & 0.23 & 0.226 & 0.244 & 0.216 \\
\hline
\end{tabular}

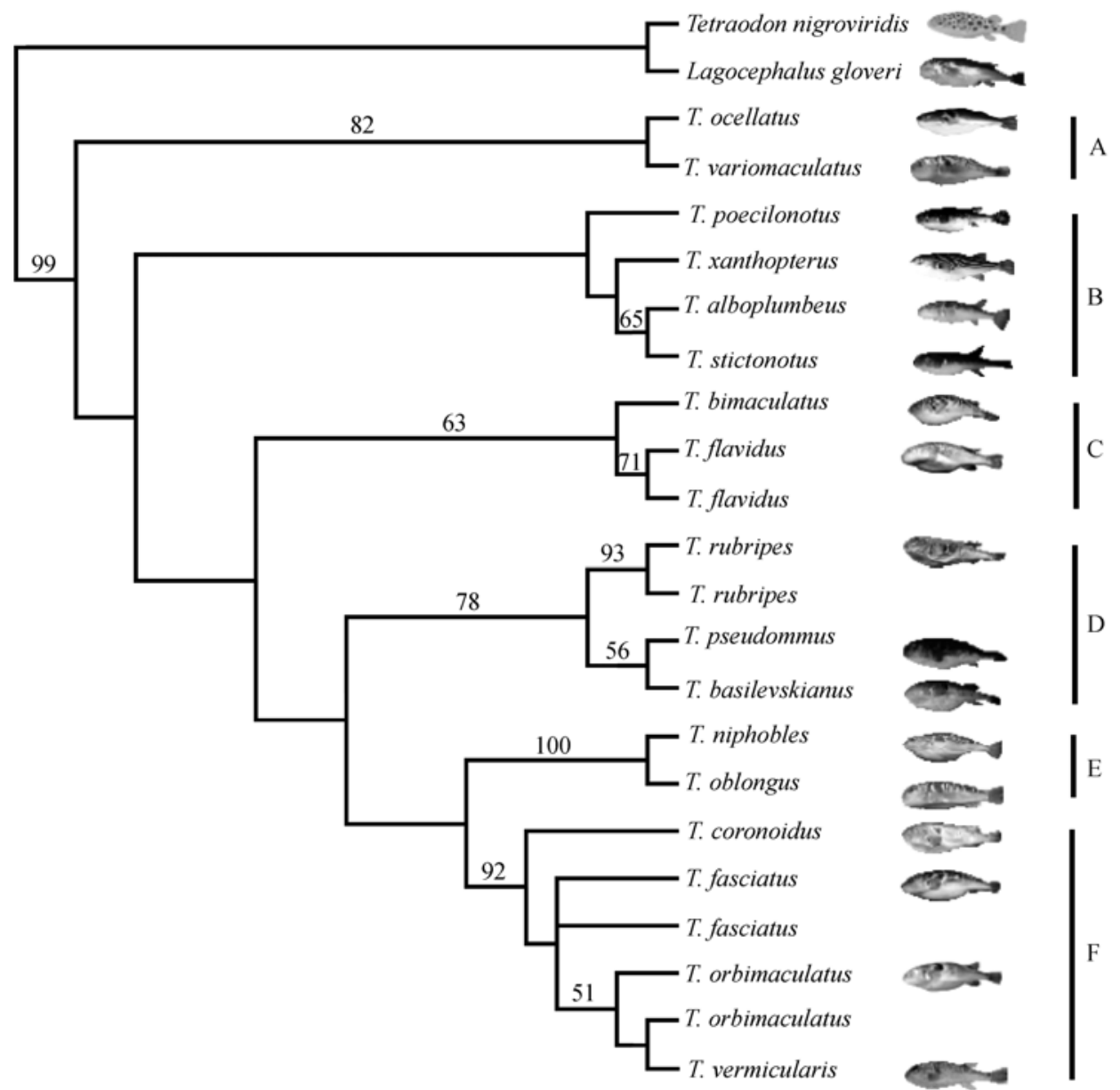

图 3 通过联合细胞色素 $b$ 基因序列和 $12 \mathrm{~S}$ rRNA 基因序列采用 GTR $+\mathrm{G}+\mathrm{I}$ 模型构建的 ML 树 $\alpha=0.5976, I=0.5458 ;-\ln L=6627.22335$, 树上的数值为自展值(低于 $50 \%$ 未展示) 
stictonotus $)(\mathrm{BS}>50)$, 其中铅点东方鲀同密点东方鲀 的关系更近 $(\mathrm{BS}=65)$; 而 $\mathrm{C}$ 支中菊黄东方鲀 $(T$. flavidus) 同双斑东方鲀 (T. bimaculatus) 的关系更近 $(\mathrm{BS}=94) ; \mathrm{D}$ 支中墨绿东方鲀 $($ T. basilevskianus $)$ 和假 睛东方鲀 (T. pseudommus)构成姐妹群关系, 然后同红 鯺东方鲀 $(T$. rubripes $)$ 一起构成一个单系类群 $(\mathrm{BS}=$ 78); E 支中星点东方鲀 (T. niphobles) 同横纹东方鲀 ( $T$. oblongus $)$ 聚在了一起 $(\mathrm{BS}=100) ; \mathrm{F}$ 支中包括有 4 个物 种, 其中晕环东方鲀 (T. coronoidus) 和暗纹东方鲀 $(T$. fasciatus) 形成姐妹群关系, 再同圆斑东方鲀 ( $T$. orbimaculatus)形成一个单系，它们一起再同虫纹东方鲀 (T. vermicularis)构成一个单系类群 $(\mathrm{BS}=92)$; 这样的 相互关系也得到其他分析方法的支持(表 3). 同时综 合各种建树方法所得到的东方鲀属鱼类的系统发育 关系, 发现 $\mathrm{D}, \mathrm{E}$ 和 $\mathrm{F}$ 支之间较其他几支而言有较近 的系统发育关系，其具体系统发育关系为 $\mathrm{F}$ 和 $\mathrm{E}$ 支构 成姐妹群关系, 它们一起再同 D 支构成一个单系群, 而 A, B 和 C 支同它们之间的关系并不明确.

表 3 MP, ME, ML 和 Bayesian 等不同建树方法的 节点支持率

\begin{tabular}{ccccc}
\hline 分支 & ML & MP & NJ & Bayesian \\
\hline A & 82 & 100 & 73 & 100 \\
B & & & & 65 \\
C & 63 & 97 & & 94 \\
D & 78 & 80 & 57 & \\
E & 100 & 100 & 94 & 100 \\
F & 92 & 98 & & 99 \\
\hline
\end{tabular}

通过不同的分析方法所得的结果发现，现有的 数据并不能完全解决该属鱼类的系统发育关系, 也 不能肯定 6 个亚类群中谁处于该属鱼类系统发育基 部的问题; 这 6 个亚类群之间的相互关系并没有得到 完全的解决。通过 Wilcoxon signed-ranks 检验和 Kishino-Hasegaw 检验对不同构树方法所得到的系统
发育树进行检验(表 4)，检验结果显示，ML，MP，NJ 和 Bayesian 等几种不同方法所得到的系统发育树的 最大似然值之间并不存在显著性差异, 这表明所获 得的几种拓扑结构间不存在显著性差异，同时两种检 验也支持了东方鲀属鱼类系一个单系类群. Buckley 等 人 ${ }^{[50]}$ 认为在这两种常用的检验方法中 ShimodairaHasegawa 检验有两种相对优势. 依据 ShimodairaHasegawa ${ }^{[46]}$ 的检验结果, 选定 ML 树作为本研究的 最优系统发育树.

由于联合基因序列中序列进化速率异质性，分 子钟假设是被拒绝的, 当克氏兔头鲀和黑青斑河豚 作为外类群时，基于细胞色素 $b$ 基因的序列对数似然 比检验表明, 分子钟约束的 ML 树似然对数值 $(-\ln L=$ 4384.71189)显著地大于无分子钟约束的似然对数值 $\left(-\ln L=4363.55237 ; \chi^{2}=42.32, \mathrm{~d} f=22, P=0.006<\right.$ 0.05). 这表明东方鲀属鱼类不同种间的细胞色素 $b$ 基 因以不同的速率进化，因此不能用细胞色素 $b$ 基因作 分子钟来估计物种的分歧时间. Peng 等人 ${ }^{[24]}$ 也发现 鮡科鱼类线粒体细胞色素 $b$ 基因有替代速率异质 现象.

\section{3 讨论}

\section{1 东方鲀属鱼类的单系性及属内系统发育关系}

东方鲀属鱼类是中国及日本邻近海洋的区域性分 布海洋鱼类，长期来有关该属内的分类及系统发育关 系比较混乱, 研究主要限于描述适应性性状及形态学 方面, 相关的分子系统学研究相对缺乏 ${ }^{[3,10,15,16]}$. 直至 1949 年, $\mathrm{Abe} \mathrm{e}^{[9]}$ 才首次定义东方鲀属鱼类为一个独立 的自然群体.

由于有关东方鲀属鱼类的研究资料相对缺乏, 本研究主要是结合同成庆泰等人 ${ }^{[15]}$ 和王可玲等人 ${ }^{[16]}$ 相关结果来探讨东方鲀属鱼类的系统发育关系的 成庆泰等人 ${ }^{[15]}$ 和王可玲等人 ${ }^{[16]}$ 的研究表明, 该属鱼

表 4 利用 Templeton ${ }^{[45]}$ 和 Shimodaira ${ }^{[46]}$ 对 MP, NJ, ML 和 Bayesian 树的检验结果 ${ }^{\text {a) }}$

\begin{tabular}{|c|c|c|c|c|c|c|c|}
\hline \multirow{2}{*}{ 树 } & \multicolumn{4}{|c|}{ Templeton 树 } & \multicolumn{3}{|c|}{ Shimodaira-Hasegawa 树 } \\
\hline & 长度 & $N$ & $Z$ & $P^{* *}$ & $-\ln L$ & $\operatorname{Diff}-\ln L$ & $P$ \\
\hline MPc 树 & 801 & 1 & -1 & 0.3173 & 6658.0538 & 10.02293 & 0.263 \\
\hline ML 树 & 801 & 24 & -0.193 & 0.8474 & 6648.0308 & 最好 & \\
\hline $\mathrm{NJ}$ 树 & 805 & 25 & -1 & 0.3173 & 6664.0564 & 16.02554 & 0.135 \\
\hline MP 树 & 800 & 最好 & & & 6654.9235 & 6.89267 & 0.397 \\
\hline Bayesian 树 & 803 & 5 & -1.342 & 0.1797 & 6655.0058 & 6.975 & 0.443 \\
\hline
\end{tabular}

a) 联合概率 $(P<0.05)$ 表明拓扑结构明显不一致, 并用*加以标明 
类是一个单系类群(图 4). 成庆泰等人 ${ }^{[15]}$ 在文中综述 了此前研究者的研究结果, 结合所获现场解剖学和 形态学等资料, 如地理分布、头骨类型、体色花纹和 体表皮刺分布等性状, 通过形态学比较, 把 15 种东 方鲀属鱼类分为了两大支，其中一大支中又包括了 6 小支(图 4(a)). 而王可玲等人 ${ }^{[16]}$ 通过肌浆蛋白的种间 差异分析, 对 13 种东方鲀属鱼类进行了聚类分析, 结果同成庆泰等人 ${ }^{[15]}$ 的存在着明显的差异(图 4(b)). 我们通过研究细胞色素 $b$ 基因和和 $12 \mathrm{~S}$ rRNA 基因序 列分析发现, 东方鲀属鱼类为一单系类群, 这同以前 的研究结果一致. 但我们在本研究中把东方鲀属鱼 类具体划分为 6 个亚类群, 这同以前研究者的结果并 不一致，且各小支中所包含的物种同以前的分析结 果也不尽相同: D 支中墨绿东方鲀和假睛东方鲀形成 姊妹群关系，在一起同红鯺东方鲀形成一个单系(BS =78), 这一结果同成庆泰等人 ${ }^{[15]}$ 结果一致, 也同 Song 等人 ${ }^{[4]}$ 研究结果一致; 而菊黄东方鲀和双斑东 方鲀形成一个姐妹群关系也同成庆泰等人 ${ }^{[15]}$ 的结果 一致, 而同王可玲等人 ${ }^{[16]}$ 的结果不一致. 本研究中发 现暗纹东方鲀同双斑东方鲀间有一个相对较近的系 统发育关系, 而他们的研究中, 菊黄东方鲀同横纹东 方鲀形成一个姐妹群关系，且双斑东方鲀同暗纹东 方鲀间的关系较近. 本研究中, 密点东方鲀和铅点东
方鲀形成姐妹群关系，再同斑点东方鲀关系较近的 黄鯺东方鲀形成一个单系群. $\mathrm{F}$ 支中包含有暗纹东方 鲀、晕环东方鲀、圆斑东方鲀和虫纹东方鲀 4 个种的 鱼. 这些结果都与成庆泰等人 ${ }^{[15]}$ 和王可玲等人 ${ }^{[16]}$ 的 研究结果不一致, 但是本研究中把东方鳋属鱼类划 分为几个不同类群的结果同以前的研究结果大体是 一致的.

以前的研究结果所使用的数据大多基于形态学 基础, 主要是基于一些特定性状, 如地理分布、头骨 类型和皮刺分布等，这些性状均大多为适应性性状， 因此常出现不一致的结果. 相比之下, 我们认为分子 数据能够提供更多的系统发育信息, 研究中所获得 的分子数据，解决了东方鲀属鱼类部分种间的系统 发育关系. 我们在研究中发现 $12 \mathrm{~S}$ rRNA 基因序列在 研究过程中不能提供足够的有效信息含量，这表明 该基因序列相对于细胞色素 $b$ 基因序列和形态数据 而言, 不适合于这个类群的系统发育分析. 本研究中, 分子系统树的一些内部节点支持率，特别是低端分 支的节点支持率较低，考虑到线粒体基因，特别是细 胞色素 $b$ 基因在种内种间等不同分类单元中可以提 供良好的系统发育结果 ${ }^{[51,52]}$, 而本研究中所采用的 线粒体分子标记在研究东方鲀属鱼类的系统发育关 系中并没有给出非常清晰的系统发育关系结果，特

(b)

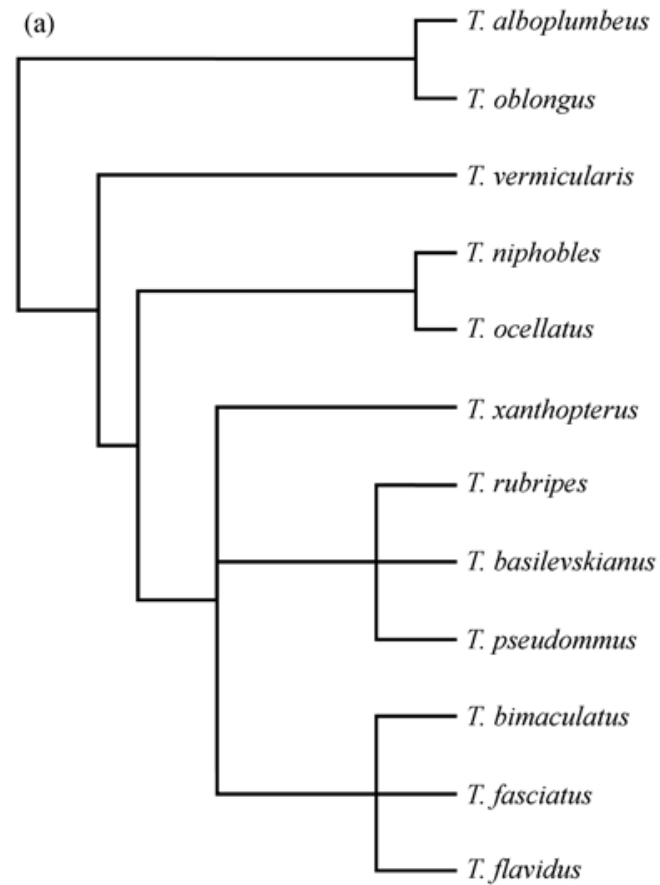

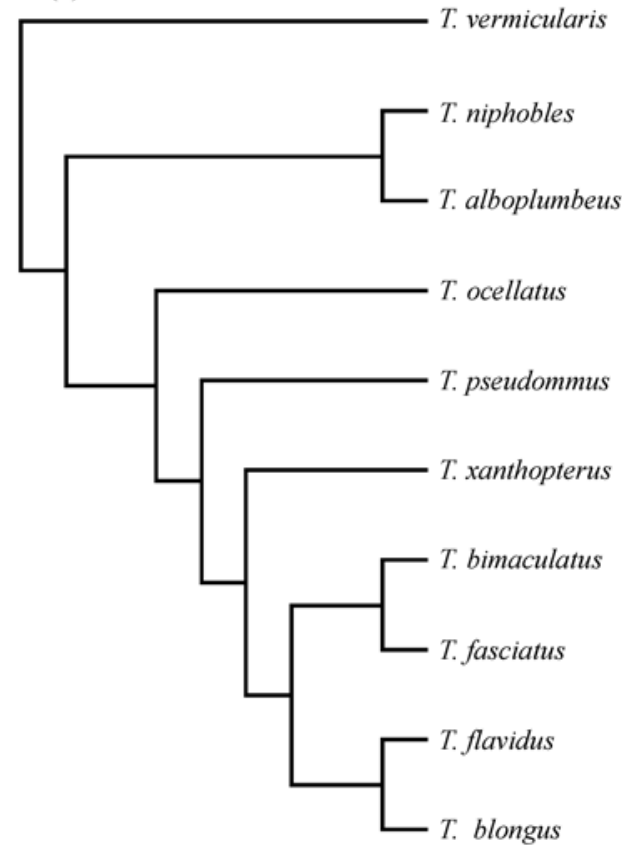

图 4 成庆泰等人 ${ }^{[15]}(\mathrm{a})$ 和王可玲等人 ${ }^{[16]}(\mathrm{b})$ 给出的聚类关系图 
别是节点内部的系统发育关系并不完全一致, 这表 明该属鱼类在分子水平上系统发育关系较为接近, 该类群形成的时间可能较短, 分化速度可能较快; 可 能反映在东方鲀属鱼类的进化历史的某些方面, 比 如在相对较短的时期内发生了多次分化事件 ${ }^{[53]}$.

\section{2 东方鲀属鱼类的物种分化和分类学含义}

鲀科鱼类起源于暖水性热带海洋水域, 随种的 分化演变, 向温冷水海域和内陆淡水水域扩散分布, 它们被认为是硬骨鱼类中最进化、最特化的类群 ${ }^{[26]}$. 而东方鲀属鱼类主要是区域分布的类群, 游泳能力 一般, 分布区域限于太平洋西海岸沿岸, 形态上主要 是通过体色花纹、体形、气囊和体表的皮刺分布与其 他类群的鱼类相区别, 大多数种都是近海岸分布的. 本研究通过分子数据, 结合生物地理方面的材料, 推 测东方鲀属鱼类的演化路线可能同鲀科鱼类中其他 类群不一致, 而且该属鱼类起源时间可能比较短而 物种分化演变速度可能比较快, 但因为相关的化石 记录缺乏, 所以需要更多的证据来阐明这个问题.

分子数据表明东方鲀属鱼类的系统发育关系非 常接近. 对比形态资料我们发现, 以前研究主要是使 用了对形态学上的一些特征的对比, 如头骨类型、地 理分布和体表皮刺分布等特征. 这些特征大多表现 为适应性性状，对应于分子数据而言可能比较主观， 也容易与分子数据相矛盾, 如铅点东方鲀和横纹东 方鲀主要基于在形态数据中的地理分布和头骨类型, 聚类中为姐妹群关系, 且同其他物种关系较远 ${ }^{[15]}$, 但 是通过遗传距离估算, 其遗传距离为 0.01 , 为属内中 等分化水平.

研究过程中, 我们注意到有些物种在形态学上 非常相似, 相互间不容易区别, 这在《中国动物志》[26] 中也有相应的记载; 但是, 分子数据结果则表明它们 之间的差距比较明显. 如星点东方鲀和菊黄东方鲀, 根据文献记载, 这两种鱼因为形态花纹都比较相似, 所以很容易混淆, 但两者之间的遗传距离还是相对 较远(5.4\%). Kadereit ${ }^{[54]}$ 指出, 适应性特征会随着基因 突变的增加而呈递增趋势, 这明显同在该属鱼类中 观察到的结果不一致. 选择上的优势或发生在小的 隔离群体上的突变产生的性状在固定下来后, 绝大 多数同由分化产生的性状不一致, 这表明一些特定 的类群(如菊黄东方鲀)在相似的环境条件下获得了 一些相类似的适应性性状, 这可能也为该类群分化
速度较快提供了依据.

成庆泰等人 ${ }^{[15]}$ 指出, 由于个体外形差异和体表 花纹比较类似, 东方鲀属鱼类的分类长期以来一直 比较混乱. 我们注意到即便是在成庆泰等人 ${ }^{[15]}$ 和 《中 国动物志》 ${ }^{[26]}$ 中被定义为独立物种的该属鱼类中, 仅 凭形态学方法也不能明显地把两物种完全分开. 而 可测量性状也可能混淆(未发表数据), 如在本研究中 所提及的晕环东方鲀和暗纹东方鲀间的遗传距离差 异较小 (近似为 0 ), 序列一致程度非常高, 而形态学 差异也非常的小. 那么关于这两个物种间是否存在 同种异名的现象是一个令人感兴趣的问题, 也需要 更多的数据加以证实.

本研究中让人感兴趣的地方还是研究过程中发 现的、与有关该属物种分化可能相关的独特的物种分 化方式. 在珠江流域采集到的弓斑东方鲀和花斑东 方鲀可能是阐述这种分化方式较好的研究材料. 我 们在采样过程中发现花斑东方鲀呈区域分布，仅在 珠江口能够找到; 而弓斑东方鲀则呈一个相对较广 的分布区域, 它们可以沿东海海岸分布至南海沿岸. 这些在《中国动物志》 ${ }^{[26]}$ 中也有记载. 这两种鱼的形 态特征非常接近, 它们之间的主要形态差异表现在 体背上的花纹, 在系统发育关系中这两种鱼也表现 为姐妹群关系. 采样过程中, 我们还发现在珠江口流 域还存在这两个种的中间型个体(未发表数据). Song 等人 ${ }^{[4]}$ 的研究表明, 同样的现象也可能存在于渤海湾. 区域种和广布种分布对应于墨绿东方鲀和红鯺东方 鲀、假睛东方鲀 ${ }^{[26]}$, 它们之间的主要差异表现为臀鯺 末端的颜色和细微的体表颜色变化. Song 等人 ${ }^{[4]}$ 的研 究指出, 在红鯺东方鲀和假睛东方鲀中广泛存在着 种间杂交现象, 中间型个体也广泛存在. 同时, 也有 研究表明黄鯺东方鲀和虫纹东方鲀间也存在着自然 杂交现象 ${ }^{[2]}$. 我们在归纳这一现象时发现, 这两个区 域存在着一些明显的共同点: (i ) 低分化程度的物 种广泛存在; (ii) 物种多样性比较丰富; (iii) 存在着 中间型个体和种间杂交的现象. 作为一个海洋类群, 区域分布、不同盐度的水体和较差的游泳能力使东方 鲀属鱼类的种间隔离成为可能, 同时也为物种分化 创造了条件. 由于特定区域存在着较多的物种, 这就 意味着种间杂交成为可能，同时也表明种间杂交可 能在东方鲀属鱼类的物种分化过程中起着很重要的 作用. 而大河口所形成的咸淡水区域也可能在东方 鲀属鱼类的物种分化过程中扮演着类似于分化中心 
这样的角色. 此外, 我们也推测这样的区域可能不是 唯一的. 这就意味着东方鲀属鱼类可能不同于鲀科 鱼类的分化主流，那么进一步研究该类群的分化方 式, 将是一个令人感兴趣的研究方向. 联系到人类自 身的进化过程, 在 Takifugu 作为模式生物扮演着越来 越重要的功能基因组时代, 深入研究该类群鱼类的 内部分化关系将在未来给予我们更多的启示.

致谢 Tulane 大学的 Mollie Cashner 博士、中国科学院水 生生物研究所的彭作刚博士、王绪祯博士和罗静博士对论 文手稿进行了修改. 中国科学院水生生物研究所的李志强 博士研究生协助采集了部分标本; 本实验室的李俊兵博士 研究生和同所的尹隽博士在实验室工作方面提供了很多有 力的帮助. 这里向他们表示最诚挚的谢意. 同时也要感谢 中国科学院海洋研究所宋林生研究员惠赠的 DNA 样本.

\section{参 考 文 献}

1 Santini F, Tyler J C. A phylogeny of the families of fossil and extant tetraodontiform fishes (Acanthomorpha, Tetraodontiformes), Upper Cretaceous to Recent. Zool J Linnean Soc, 2003, 139(4): 565-617[DOI]

2 Masuda Y, Shinohara N, Takahashi Y, et al. Occurrence of natural hybrid between pufferfishes, Takifugu xanthopterus and T. vermicularis, in Ariake Bay, Kyushu, Japan. Nippon Suisan Gakkaishi, 1991, 57(7): 1247-1255

3 Masuda H, Amaoka K, Araga C, et al. The fishes of the Japanese Archipelago, vol. Text and Plates. Tokyo: Tokai Univ Press. 1984

4 Song L, Liu B, Xiang J, et al. Molecular phylogeny and species identification of pufferfish of the genus Takifugu (Tetraodontiformes, Tetraodontidae). Mar Biotechnol (NY), 2001, 3(4): 398406[DOI]

5 Aparicio S, Chapman J, Stupka E, et al. Whole-genome shotgun assembly and analysis of the genome of Fugu rubripes. Science, 2002, 297(5585): 1301-1310[DOI]

6 Brenner S, Elgar G, Sandford R, et al. Characterization of the pufferfish (Fugu) genome as a compact model vertebrate genome. Nature, 1993, 366(6452): 265-268[DOI]

7 Elmerot C, Arnason U, Gojobori T, et al. The mitochondrial genome of the pufferfish, Fugu rubripes, and ordinal teleostean relationships. Gene, 2002, 295(2): 163-172[DOI]

8 Fraser-Brunner A. Notes on the plectognath fishes. VIII. The classification of the suborder Tetraodontoidea, with a synopsis of the genera. Ann Mag Nat Hist, 1943, 10(61): 1-18

9 Abe T. Taxonomic studies on the puffers (Tetraodontidae, Teleostei) from Japan and adjacent regions, $V$ : Synopsis of the puffers from and adjacent regions. Bull Biogeogr Soc Japan, 1949, 14(1, 13): 1 $-15,89-140$, pls. $141-142$

10 Abe T. Taxonomic studies on the puffers (Tetraodontidae, Teleostei) from Japan and adjacent regions, VII: Concluding remarks, with the introduction of two new genera, Fugu and Boesemanichthys. Jpn J Ichthyol, 1952, 2(1, 2, 3): 35—44; figs. 31-33, 93-97, 117 $-127$

11 Whitley G P. Studies in ichthyology. Rec Aus Mus, 1953, 23: 133 $-138$

12 Holcroft N I. A molecular test of alternative hypotheses of tetraodontiform (Acanthomorpha: Tetraodontiformes) sister group relationships using data from the RAG1 gene. Mol Phylogenet Evol, 2004, 32(3): 749-760[DOI]

13 Holcroft N I. A molecular analysis of the interrelationships of tetraodontiform fishes (Acanthomorpha: Tetraodontiformes). Mol Phylogenet Evol, 2005, 34(3): 525-544[DOI]

14 Chen W J, Orti G, Meyer A. Novel evolutionary relationship among four fish model systems. Trends Genet, 2004, 20(9): 424431[DOI]

15 成庆泰, 王存信, 田明诚, 等. 中国东方鲀属鱼类分类研究. 动 物学报, 1975, 21(4): 359-378

16 王可玲, 张培军, 尹青. 东方鲀属鱼类肌浆蛋白的种间差异及其 聚类的分析. 海洋与湖沼, 1984, 15(5): 493-499

17 Miyaki K, Tebeta O, Kayano H. Karyotypes in six species of pufferfishes genus Takifugu (Tetraodontidae, Tetraodontiformes). Fish Sci, 1995, 61: 594-598

18 陈超, 石拓, 孙曙光, 等. 应用 RAPD 标记对东方鲀属进行种类 鉴别及其聚类分析. 海洋水产研究, 2001, 22(3): 32-36

19 Song L, Liu B, Wang Z, et al. Phylogenetic relationships among pufferfish of genus Takifugu by RAPD analysis. Chin J Oceanol Limnol, 2001, 19(2): 128-134

20 Song L, Li H, Cui Z, et al. Population genetic structure and genetic differentiation of the pufferfish Takifugu rubripes and Takifugu pseudommus revealed by RAPD Analysis. High Technol Lett, 2003, 9(1): $22-25$

21 Esposti M D, de Vries S, Crimi M, et al. Mitochondrial cytochrome b: evolution and structure of the protein. Biochim Biophys Acta, 1993, 1143(3): 243-271[DOI]

22 Miya M, Nishida M. Use of mitogenomic information in teleostean molecular phylogenetics: A tree-based exploration under the maximum-parsimony optimality criterion. Mol Phylogenet Evol, 2000, 17(3): 437-455[DOI]

23 Zardoya R, Meyer A. Phylogenetic performance of mitochondrial protein-coding genes in resolving relationships among vertebrates. Mol Biol Evol, 1996, 13(7): 933-942

24 Peng Z, He S, Zhang Y. Phylogenetic relationships of glyptosternoid fishes (Siluriformes: Sisoridae) inferred from mitochondrial cytochrome b gene sequences. Mol Phylogenet Evol, 2004, 31(3): 979-987[DOI]

25 崔建洲, 申雪艳, 杨官品, 等. 红鯺东方鲀与假睛东方鲀的微卫 星 DNA 多态性分析. 高技术通讯, 2005, 15(12): 90-96

26 苏锦祥, 李春生. 中国动物志. 北京: 科学出版社, 2002 
27 Sambrook J, Fritsch E F, Maniatis T. Molecular Cloning: A Laboratory Manual. 2nd. New York: Cold Spring Harbor Laboratory Press, 1989

28 Xiao W, Zhang Y, Liu H. Molecular systematics of Xenocyprinae (Teleostei: Cyprinidae): Taxonomy, biogeography, and coevolution of a special group restricted in East Asia. Mol Phylogenet Evol, 2001, 18(2): 163-173[DOI]

29 刘焕章. 用 mtDNA $12 \mathrm{~S}$ rRNA 序列变异检验鲤形目鱼类系统发 育关系. 遗传学报, 2004, 31(2): 137-142

30 Thompson J D, Gibson T J, Plewniak F, et al. The CLUSTAL_X windows interface: Flexible strategies for multiple sequence alignment aided by quality analysis tools. Nucleic Acids Res, 1997, 25(24): 4876-4882[DOI]

31 Galtier N, Gouy M, Gautier C. SEAVIEW and PHYLO_WIN: Two graphic tools for sequence alignment and molecular phylogeny. Comput Appl Biosci, 1996, 12(6): 543—548

32 Wiens J J. Combining data sets with different phylogenetic histories. Syst Biol, 1998, 47(4): 568-581[DOI]

33 Swofford D L. PAUP*. Phylogenetic Analysis Using Parsimony (*and other methods), Version 4th ed. Sunderland, Massachusetts: Sinauer Associates, 2002

34 Farris J S, Kallersjo M, Kluge A G, et al. Testing significance of incongruence. Cladistics, 1995, 10(3): 315-319[DOI]

35 Xia X, Xie Z. DAMBE: Software package for data analysis in molecular biology and evolution. J Hered, 2001, 92(4): $371-$ $373[\mathrm{DOI}]$

36 Tamura K, Nei M. Estimation of the number of nucleotide substitutions in the control region of mitochondrial DNA in humans and chimpanzees. Mol Biol Evol, 1993, 10(3): 512-526

37 Kumar S, Tamura K, Nei M. MEGA3: Integrated software for molecular evolutionary genetics analysis and sequence alignment. Brief Bioinformatics, 2004, 5(2): 150-163[DOI]

38 Huelsenbeck J P, Ronquist F. MRBAYES: Bayesian inference of phylogenetic trees. Bioinformatics, 2001, 17(8): 754-755[DOI]

39 Farris J S. Methods for computing Wagner trees. Syst Zool, 1970, 19(1): $83-92[\mathrm{DOI}$

40 Felsenstein J. Evolutionary trees from DNA sequences: A maximum likelihood approach. J Mol Evol, 1981, 17(6): $368-$ 376[DOI]

41 Mau B. Bayesian phylogenetic inference via Markov chain Monte
Carlo methods. Madison: University of Wisconsin-Madison, 1996

42 Felsenstein J. Confidence limits on phylogenies: An approach using the bootstrap. Evolution, 1985, 39(4): 783-791[DOI]

43 Posada D, Crandall K A. MODELTEST: Testing the model of DNA substitution. Bioinformatics, 1998, 14(9): 817-818[DOI]

44 Lanave C, Preparata G, Saccone C, et al. A new method for calculating evolutionary substitution rates. J Mol Evol, 1984, 20(1): 86 $-93[\mathrm{DOI}]$

45 Templeton A R. Phylogenetic inference from restriction endonuclease cleavage site maps with particular reference to the evolution of humans and the apes. Evolution, 1983, 37(2): 221-244[DOI]

46 Shimodaira H, Hasegawa M. Multiple comparisons of loglikelihoods with applications to phylogenetic inference. Mol Biol Evol, 1999, 16(8): 1114-1116

47 Irwin D M, Kocher T D, Wilson A C. Evolution of the cytochrome $b$ gene of mammals. J Mol Evol, 1991, 32(2): 128-144[DOI]

48 Lydeard C, Roe K J. The phylogenetic utility of the mitochondrial Cytochrome $\mathrm{b}$ gene for inferring relationships among Actinopterygian fishes. San Diego: Academic Press, 1997

49 Cantatore P, Roberti M, Pesole G, et al. Evolutionary analysis of cytochrome $b$ sequences in some Perciformes: Evidence for a slower rate of evolution than in mammals. J Mol Evol, 1994, 39(6): $589-597[\mathrm{DOI}]$

50 Buckley T R, Simon C, Shimodaira H, et al. Evaluating hypotheses on the origin and evolution of the New Zealand alpine cicadas (Maoricicada) using multiple-comparison tests of tree topology. Mol Biol Evol, 2001, 18(2): 223-234

51 Near T J, Porterfield J C, Page L M. Evolution of Cyt $b$ and the molecular systematics of Ammocrypta (Percidae: Etheostomatinae). Copeia, 2000, 3: 701-711[DOI]

52 Johnson J B, Jordan S. Phylogenetic divergence in leatherside chub (Gila copei) inferred from mitochondrial cytochrome $b$ sequences. Mol Ecol, 2000, 9(8): 1029-1035[DOI]

53 Guo X, He S, Zhang Y. Phylogeny and biogeography of Chinese sisorid catfishes re-examined using mitochondrial cytochrome b and 16S rRNA gene sequences. Mol Phylogenet Evol, 2005, 35(2): $344-362[\mathrm{DOI}]$

54 Kadereit J W. Molecules and morphology, phylogenetics and genetics. Bot Acta, 1994, 107: 369-373 